\title{
Spectral analysis of high resolution near-infrared spectra of ultra cool dwarfs
}

\author{
Y. Lyubchik ${ }^{1}$, H. R. A. Jones ${ }^{2}$, Y. V. Pavlenko ${ }^{1,2}$, E. Martin ${ }^{3,4}$, I. S. McLean ${ }^{5}$, L. Prato ${ }^{6}$, R. J. Barber ${ }^{7}$, and J. Tennyson ${ }^{7}$ \\ 1 Main Astronomical Observatory of Academy of Sciences of Ukraine, Zabolotnoho, 27, Kyiv 03680, Ukraine \\ e-mail: lyu@mao.kiev.ua \\ 2 Center for Astrophysics Research, University of Hertfordshire, College Lane, Hatfield, Hertfordshire AL10 9AB, UK \\ 3 Instituto de Astrofísica de Canarias, La Laguna, Tenerife 38200, Spain \\ ${ }^{4}$ University of Central Florida, Department of Physics, PO Box 162385, Orlando, FL 32816, USA \\ 5 Department of Physics and Astronomy, UCLA, Los Angeles, CA 90095, USA \\ ${ }^{6}$ Lowell Observatory, 1400 West Mars Hill Road, Flagstaff, AZ 86001, USA \\ 7 Department of Physics and Astronomy, University College London, Gower Street, London WC1E 6BT, UK
}

Received 7 April 2006 / Accepted 11 July 2007

\section{ABSTRACT}

\begin{abstract}
Aims. We present an analysis of high resolution spectra in the $J$ band of five ultra cool dwarfs from M6 to L0. Methods. We make use of a new ab initio water vapour line list and existing line lists of $\mathrm{FeH}$ and $\mathrm{CrH}$ for modelling the observations. Results. We find a good fit for the Mn I $12899.76 \AA$ line. This feature is one of the few for which we have a reliable oscillator strength. Other atomic features are present but most of the observed features are $\mathrm{FeH}$ and $\mathrm{H}_{2} \mathrm{O}$ lines. While we are uncertain about the quality of many of the atomic line parameters, the $\mathrm{FeH}$ and $\mathrm{CrH}$ line lists predict a number of features which are not apparent in our observed spectra. We infer that the main limiting factor in our spectral analysis is the $\mathrm{FeH}$ and $\mathrm{CrH}$ molecular spectra.
\end{abstract}

Key words. line: identification - stars: atmospheres - stars: low-mass, brown dwarfs - stars: late-type

\section{Introduction}

Observations show that $\mathrm{M}$ dwarfs are the most numerous population in the solar neighbourhood. The total number of very low mass stars and cool objects, which include $\mathrm{L}$ and $\mathrm{T}$ dwarfs, could be much higher, e.g., from simulations (Allen et al. 2005) or from inferences from nearby star counts (Henry et al. 2005).

Molecular absorption plays the definitive role in the appearance of ultra cool dwarf spectra. Molecular bands govern the overall shape of the infrared observed spectra of cool dwarfs. In spite of a lot of effort in measuring and computing more accurate and full molecular line lists the situation is far from ideal. This is caused by a lack of accurate oscillator strengths and quantummechanical data for molecules in the infrared region.

The situation is not ideal even for atomic lines which in comparison to molecular lines are relatively straightforward to obtain. Most of the atomic lines in the infrared are formed by transitions between upper levels of atoms which are difficult to compute with high accuracy. As shown in Lyubchik et al. (2004), strong atomic lines in the spectra of ultra cool dwarfs can be identified even against a background of molecular absorption. Comparison of these atomic lines with computed profiles can be used as key diagnostics of ultra cool dwarf atmospheres.

The peak of the energy distribution for most cool and ultra cool dwarfs is located in or close to the $J$ band. Many authors have studied the $J$-band region of ultra cool dwarfs. Kirkpatrick et al. (1993) and Jones et al. (1994, 1996) provided identifications of spectral features and indicated it as a productive spectral region to study. Molecular absorption in the $J$ band is comparatively weak, so atomic lines of even intermediate strength are easily identified. The advent of the newest infrared spectrographs mean that good quality high resolution spectra can be taken of brown dwarfs and ultra cool dwarfs (e.g., see McLean et al. 2007; Zapatero Osorio et al. 2006, and spectra shown in our paper). However, despite these advantages for spectral analysis the determination of the physical parameters has so far relied only on the strongest lines (e.g., see Leggett et al. 2001).

\section{Observational data}

The observational data details and characteristics of the target stars are presented in Table 1. Hereafter we use the following designations: $2 \mathrm{M} 0140+27$ for $2 \mathrm{MASSW}$ J0140026 +270150 and $2 \mathrm{M} 0345+25$ for $2 \mathrm{MASP} \mathrm{J} 0345432+254023$. The spectra are shown in Fig. 1.

The spectra were obtained with NIRSPEC on Keck. The reduction of these spectra was carried out according to standard procedures (McLean et al. 2003; Prato et al. 2002a,b; Zapatero Osorio et al. 2006). Integration times ranged from $10 \mathrm{~min}$ to $1.5 \mathrm{~h}$ depending on $J$-band magnitude. For the faintest objects, the individual exposures were $600 \mathrm{~s}$, for brighter objects $-300 \mathrm{~s}, 120 \mathrm{~s}$, or 60 s (see McLean et al. 2007; and Zapatero Osorio et al. 2006, for details).

For all stars we use the 59 NIRSPEC echele order (12 826-13015 $\AA$, see Table 2 in McLean et al. 2007). The resolving power of the spectra is $R \sim 20000$ (it varies from $R=17800$ at $11480 \AA$ to $R=22700$ at $13460 \AA$ ). SNRs are in the range of $\sim 50$ for $2 \mathrm{M} 0345+25$ to over 100 for GJ 406 and $2 \mathrm{M} 0140+27$.

We note the presence of $\mathrm{OH}$ night sky emission lines though at the relatively high-resolution $(R \sim 20000)$ used here they 
Table 1. List of programme stars including their spectral type (based on optical spectroscopy), effective temperatures and $v \sin i$ (with references in brackets).

\begin{tabular}{cccc}
\hline \hline Name & Spectral type & $T_{\text {eff }}$ & $v \sin i, \mathrm{~km} \mathrm{~s}^{-1}$ \\
\hline GJ 406(LHS 36) & M6 V (K91) & $2800 \mathrm{~K}(\mathrm{~B} 00)$ & $3(\mathrm{MB} 03)$ \\
VB 10 (LHS 474) & M8 V (K91) & $2700 \mathrm{~K}(\mathrm{~S} 96)$ & $\sim 10(\mathrm{J05})$ \\
2MASSW J0140026+270150 & M8.5 V (Gi00) & $2500 \mathrm{~K}$ & $6.5(\mathrm{R} 02)$ \\
LP 944-20 (2MASSW J0339352-352544) & M9 V(K99)/M9.5 V(R02) & $2400 \mathrm{~K}(\mathrm{~B} 00)$ & $\sim 30(\mathrm{TR} 98)$ \\
2MASP J0345432+254023 & L0 (K99) & $2200 \mathrm{~K}(\mathrm{~B} 00)$ & $45(*)$ \\
\hline
\end{tabular}

B00 - Basri et al. (2000); Gi00 - Gizis et al. (2000); J05 - Jones et al. (2005); K91 - Kirkpatrick et al. (1991); K99 - Kirkpatrick et al. (1999); MB03 - Mohanty \& Basri (2003); R02 - Reid et al. (2002); S96 - Schweitzer et al. (1996); TR98 - Tinney \& Reid (1998); (*) determined using spectra fitting (see Sect. 4.4).

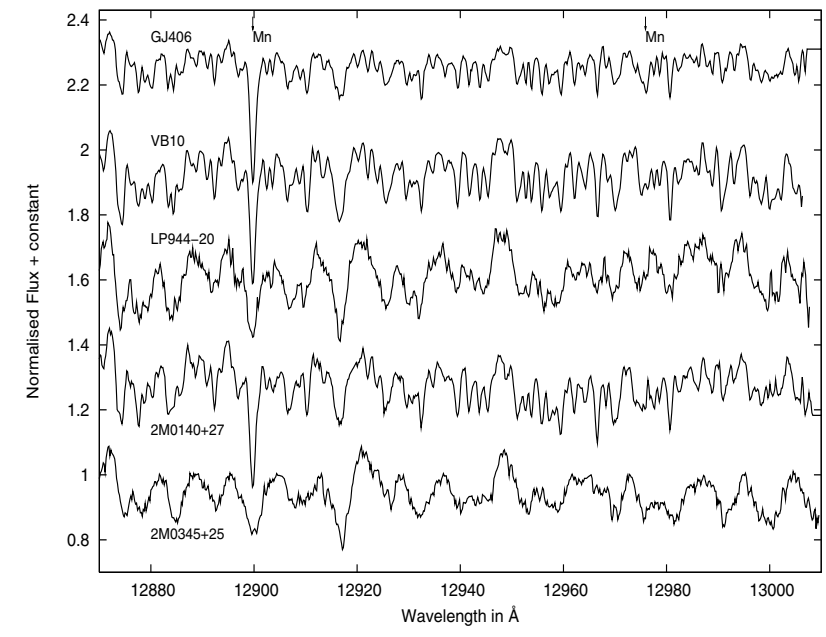

Fig. 1. Spectral sequence of the targets in spectral type order. The positions of $12899.76 \AA$ and $12975.91 \AA$ Mn I lines are shown.

are not strong enough to saturate the detector. If the $\mathrm{OH}$ line strengths have changed in two subsequent exposures, the reduction software, REDSPEC, removes any residual OH night sky lines (see McLean et al. 2007, for details).

Our targets are stars with non-zero radial velocities (e.g., see Martin et al. 2006). We shifted the spectra to zero velocity using the Mn I $12899 \AA$ line which is a prominent feature in all spectra.

\section{Procedure}

\subsection{Synthetic spectra modelling}

Synthetic spectra are computed using the WITA6 programme (Pavlenko 2000) for NextGen model structures (Hauschildt et al. 1999). Calculations are carried out under the assumption of local thermodynamic equilibrium, hydrostatic equilibrium, in the absence of sources and sinks of energy. The atomic line list used for our spectral modeling and line identification is taken from the VALD database (Kupka et al. 1999). The solar abundances reported by Anders \& Grevesse (1989) are used in calculations. All details of other input parameters are described by Pavlenko et al. (2000).

To compute the synthetic spectra we used molecular line lists from different sources discussed below. The relative contributions of these molecules to the formation of the spectra are shown in Fig. 2. Theoretical spectra are computed with a wavelength step of $0.01 \AA$ and convolved with Gaussians to match the instrumental broadening. We adopt a $F W H M$ of $1.2 \AA$. The

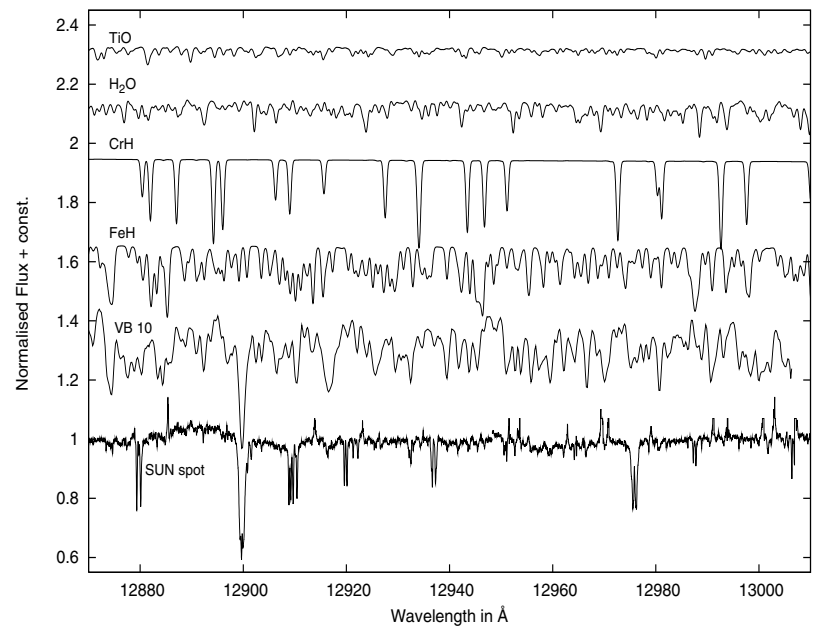

Fig. 2. Spectral sequence of observed spectra of Solar umbra, VB10 and synthetic calculations for $\mathrm{FeH}, \mathrm{CrH}, \mathrm{H}_{2} \mathrm{O}$ (BT2 line list) and $\mathrm{TiO}$ molecules across the region of interest.

rotational broadening of spectral lines is implemented following Gray (1976).

\subsubsection{Water vapour line lists}

Water vapour is the main contributor to the opacity across most of the infrared for late type $\mathrm{M}$ dwarfs and brown dwarfs. There are several $\mathrm{H}_{2} \mathrm{O}$ line lists which are used in computations of synthetic spectra of dwarfs: HITRAN (Rothman et al. 1992), MT (Miller et al. 1994), AMES (Partridge \& Schwenke 1997), SCAN (Jrgensen et al. 2001) and VT2 (Viti et al. 1997). For details and comparisons of these line lists the reader is referred to Pavlenko (2002) and Jones et al. (2002, 2003, 2005). In our computations we used the AMES and BT2 line lists. We give a short description of each line list.

The AMES (Partridge \& Schwenke 1997) water vapour line list was computed using a potential energy surface (Murrell et al. 1984) which was fitted to line frequencies with $J<5$, given in the HITRAN (Rothman et al. 1992, http: //www . hitran. com) database. The line intensities are calculated using an ab initio dipole moment surface. It has helped enable a wide range of photometric and spectroscopic results.

The BT2 synthetic line list for $\mathrm{H}_{2} \mathrm{O}$ (Barber et al. 2006) was computed using a discrete variable representation implemented within the DVR3D suite of programs (Tennyson et al. 2004) to calculate the rotation-vibration spectra of triatomic molecules. BT2 also used a potential energy surface obtained by fitting to laboratory spectra (Shirin et al. 2003) and an ab initio dipole surface (Schwenke \& Partridge 2000). It is the most complete 
water line list, comprising over 500 million transitions $(65 \%$ more than any other list) and is the most accurate one (over $90 \%$ of all known experimental energy levels are within $0.3 \mathrm{~cm}^{-1}$ of the BT2 values). Tests by Barber et al. (2006) show that at $3000 \mathrm{~K}, \mathrm{BT} 2$ includes $99.2 \%$ of the water opacity in the $J$ band region, which represents a halving of the (1.6\%) opacity missing in the previously best-available list, AMES (Partridge \& Schwenke 1997). Nonetheless our analysis is concerned with objects where the temperatures of the line forming regions are less than $3000 \mathrm{~K}$ and so it is perhaps unsurprising that our analysis is insensitive to the differences in synthetic spectra computed using AMES and BT2 $\mathrm{H}_{2} \mathrm{O}$ line lists. Here we show the spectra computed using the BT2 line list.

\subsubsection{Other molecules}

Molecules of $\mathrm{FeH}$ and $\mathrm{CrH}$ are also important opacity sources in the spectra of ultra cool dwarfs. In our calculations we used line lists of FeH (Dulick et al. 2003) and CrH (Burrows et al. 2002).

The line list of $\mathrm{FeH}$ (Dulick et al. 2003) pertains to the $F^{4} \Delta_{i}-X^{4} \Delta_{i}$ transitions using the spectroscopic constants of rotational levels $(v=0,1,2)$ of the FeH X and F states. Dulick et al. extrapolate for values $v=3,4$ and $J$-values up to 50.5. The line list of $\mathrm{FeH}$ we use then consists of experimental and extrapolated term values for the 25 vibrational bands with $v \leq 4$. In this line list ${ }^{54} \mathrm{Fe},{ }^{57} \mathrm{Fe}$ and ${ }^{58} \mathrm{Fe}$ along with the most abundant isotope ${ }^{56} \mathrm{Fe}$ (91.7\% of iron abundance) are accounted for.

The $\mathrm{CrH}$ line list (Burrows et al. 2002) contains information on lines of 12 bands of the $A^{6} \Sigma^{+}-X^{6} \Sigma^{+}$electronic system and includes ground state term values for vibrational levels $v=0$, 1,2 up to $J=39.5$. For terms with $v=3$ and high $J$, Burrows et al. extrapolate from Bauschlicher et al. (2001). The most abundant ${ }^{52} \mathrm{Cr}$ isotope $\left(83.3 \%\right.$ of chromium abundance), ${ }^{50} \mathrm{Cr},{ }^{53} \mathrm{Cr}$ and ${ }^{54} \mathrm{Cr}$ are also included in $\mathrm{CrH}$ line list.

We include TiO absorption (Plez 1998) in our spectral analysis though $\mathrm{TiO}$ is not a significant contributor at these wavelengths (see Fig. 2). We note, that another $\mathrm{TiO}$ line list by Schwenke (1998) may be used to account for TiO absorption. However, Pavlenko et al. (2006a) indicates that both sources will provide similar opacity over this spectral region.

\section{Results}

We compute the individual contributions of the atomic and molecular species for the wavelength region 12800-13010 (Fig. 2). While $\mathrm{TiO}$ and $\mathrm{H}_{2} \mathrm{O}$ do not show strong lines in the region, their numerous absorption lines (especially $\mathrm{H}_{2} \mathrm{O}$ ) form the pseudo-continuum above $2500 \mathrm{~K}$. As the effective temperature drops below $2500 \mathrm{~K}$ the contribution to the total opacity by $\mathrm{FeH}$ and $\mathrm{CrH}$ becomes dominant.

In Fig. 3 we show the comparison of synthetic spectra $2800 / 5.0 / 0.0\left(T_{\text {eff }}=2800 \mathrm{~K}, \log g=5.0\right.$ and $\left.[\mathrm{M} / \mathrm{H}]=0.0\right)$ and 2500/5.0/0.0, computed using $\mathrm{TiO}, \mathrm{CrH}, \mathrm{FeH}$ and $\mathrm{H}_{2} \mathrm{O}$ (BT2) molecular line lists, with the observed GJ406 (panel a) and 2M0140+27 (panel b) spectra.

$\mathrm{CrH}$ lines can be identified in the observed spectra (see Table 2). However, analysis of the observed $\mathrm{CrH}$ spectrum is very problematic due to the high multiplicity of the upper electronic state of the A-X transition of CrH (Burrows et al. 2002). From comparison of Figs. 3a,b, 4 and 6 we find the use of the $\mathrm{CrH}$ opacity causes a poorer fit both for GJ406 and 2M0140+27. From Fig. 3 onward we show the synthetic spectra computed without the $\mathrm{CrH}$ line list.

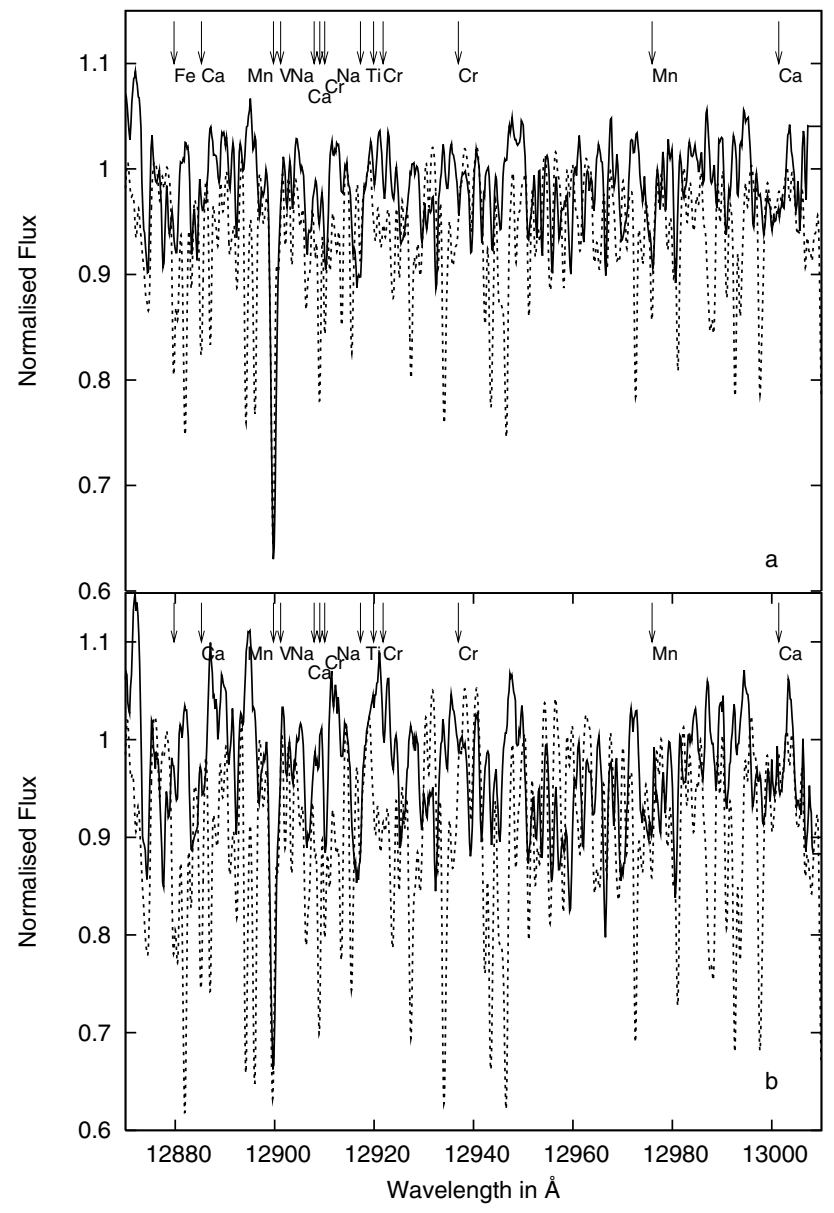

Fig. 3. a) The observed GJ406(M6 V) and b) $2 \mathrm{M} 0140+27(\mathrm{M} 8.5 \mathrm{~V}$ ) spectra (solid line) compared to the 2800/5.0/0.0 and 2500/5.0/0.0 synthetic spectra, computed using molecular line lists of $\mathrm{H}_{2} \mathrm{O}(\mathrm{BT} 2)$, TiO, $\mathrm{FeH}, \mathrm{CrH}$ (dotted line).

We compute a set of synthetic spectra for model atmospheres of different effective temperatures appropriate to our cool dwarfs. Assuming our observed stars are dwarfs, we adopt $\log g=5.0$ for all computations except for LP944-20 where we used a value of $\log g=4.5$ (Pavlenko et al. 2006b). We find the differences between synthetic spectra computed for models of $\Delta \log g= \pm 0.25$ and $[\mathrm{M} / \mathrm{H}]= \pm 0.2$ are rather marginal. Given the lack of evidence for non-Solar metallicities in the literature for our target stars, we assume Solar metallicities.

Our choices of effective temperatures are based on the spectral type estimations by different authors (see Table 1). Golimowski et al. (2004) provides effective temperature ranges depending on the age of stars. For the hottest star from our program list GJ 406 and the coolest star $2 \mathrm{M} 0345+25$ Golimowski et al. give the ranges of $T_{\text {eff }}=2650-2900 \mathrm{~K}$ and $T_{\text {eff }}=2000-2350 \mathrm{~K}$, for assumed ages of $0.1-10 \mathrm{Gyr}$ and known parallax uncertainties. The fit of the strong Mn features is consistent with the mid-point of these effective temperatures to within $100 \mathrm{~K}$.

\subsection{GJ $406 \&$ VB10}

A comparison of the observed spectrum of GJ 406 (M6 V) and a synthetic spectrum 2800/5.0/0.0 with $v \sin i \sim 3 \mathrm{~km} \mathrm{~s}^{-1}$ (e.g., Mohanty \& Basri 2003) is shown in Fig. 4. Figure 5 shows the compares the observed spectrum of VB $10(\mathrm{M} 8 \mathrm{~V})$ to a 
Table 2. Line identifications and strengths for the programme stars GJ406, VB10 and 2M0140+27 are tabulated.

\begin{tabular}{|c|c|c|c|c|c|}
\hline $\mathrm{WL} \pm 0.1 \AA ̊$, air & GJ406 & VB10 & $2 \mathrm{M} 0140+27$ & Sunspot & Notes \\
\hline 12874.4 & $* *$ & $* * *$ & $* * *$ & $* *$ & $\mathrm{FeH}, \mathrm{H}_{2} \mathrm{O}(?)$ \\
\hline 12877.7 & $* *$ & $* * *$ & $* * *$ & $*(w k)$ & $\mathrm{FeH}(?), \mathrm{TiO}(w k, ?), \mathrm{H}_{2} \mathrm{O}(?)$ \\
\hline 12878.9 & $* *$ & $* *$ & $* *$ & - & $\mathrm{H}_{2} \mathrm{O}$ \\
\hline 12879.8 & - & - & - & $* * *(\mathrm{spl})$ & $\mathrm{Fe}(\mathrm{VALD})$ \\
\hline 12880.4 & $* *$ & $* * *$ & * & - & $\mathrm{H}_{2} \mathrm{O}, \mathrm{FeH}(\mathrm{w}), \mathrm{CrH}(\mathrm{w})$ \\
\hline 12883.5 & $* *$ & $* * *$ & $* * *$ & ** & $\mathrm{FeH}, \mathrm{TiO}(w \mathrm{k})$ \\
\hline 12884.4 & $* *$ & $* * *$ & $* *(\mathrm{bl})$ & - & $\mathrm{FeH}(\mathrm{w}), \mathrm{H}_{2} \mathrm{O}(\mathrm{wk})$ \\
\hline 12885.3 & - & $* *$ & - & - & $\mathrm{Ca}(\mathrm{VALD})$ \\
\hline 12885.9 & $*$ & $* *$ & $*$ & - & $\mathrm{FeH}(\mathrm{w}), \mathrm{H}_{2} \mathrm{O}$ \\
\hline 12888.8 & * & * & * & * & $\mathrm{FeH}, \mathrm{H}_{2} \mathrm{O}$ \\
\hline 12891.0 & $*$ & $*$ & $*$ & * & $\mathrm{FeH}, \mathrm{H}_{2} \mathrm{O}$ \\
\hline 12892.4 & $* *$ & $* * *$ & $* * *$ & $* *$ & $\mathrm{H}_{2} \mathrm{O}, \mathrm{FeH}, \mathrm{TiO}(w \mathrm{k})$ \\
\hline 12893.6 & $*$ & $*$ & $*$ & - & $\mathrm{CrH}(\mathrm{w}), \mathrm{FeH}(\mathrm{w})$ \\
\hline 12897.0 & $*$ & $* *$ & $* *$ & $*$ & $\mathrm{H}_{2} \mathrm{O}(?)$ \\
\hline 12897.7 & $*$ & $*$ & $*$ & $*$ & $\mathrm{FeH}, \mathrm{H}_{2} \mathrm{O}(?)$ \\
\hline 12899.8 & $* * *$ & $* * *$ & $* * *$ & $* * *$ & Mn (VALD) \\
\hline 12902.6 & $*$ & $* *$ & $*$ & $*$ & $\mathrm{H}_{2} \mathrm{O}(?), \mathrm{TiO}(w \mathrm{k})$ \\
\hline 12903.6 & * & $* *$ & * & $* *$ & $\mathrm{FeH}, \mathrm{H}_{2} \mathrm{O}(?)$ \\
\hline 12906.5 & $* *$ & $* * *$ & ** & - & $\mathrm{H}_{2} \mathrm{O}, \mathrm{CrH}(\mathrm{w}), \mathrm{FeH}(\mathrm{w}), \mathrm{TiO}(\mathrm{wk})$ \\
\hline 12907.3 & * & $* *$ & ** & * & $\mathrm{FeH}, \mathrm{H}_{2} \mathrm{O}$ \\
\hline 12907.9 & - & - & - & - & $\mathrm{Na}(\mathrm{VALD})$ \\
\hline 12909.1 & $*$ & $* *$ & - & $* * *(\mathrm{spl})$ & $\mathrm{Ca}, \mathrm{CrH}, \mathrm{FeH}, \mathrm{H}_{2} \mathrm{O}, \mathrm{TiO}$ (wk) \\
\hline 12910.1 & - & - & - & $* * *(\mathrm{spl})$ & $\mathrm{Cr}(\mathrm{VALD})$ \\
\hline 12910.4 & $* *$ & $* * *$ & $* * *$ & $*(\mathrm{bl})$ & $\mathrm{FeH}, \mathrm{H}_{2} \mathrm{O}$ \\
\hline 12913.5 & $*$ & $*$ & $*$ & - & $\mathrm{FeH}, \mathrm{H}_{2} \mathrm{O}(?)$ \\
\hline 12916.5 & $* * *$ & $* * *$ & $* * *$ & $* *$ & $\mathrm{H}_{2} \mathrm{O}, \mathrm{FeH}(\mathrm{w}), \mathrm{TiO}(\mathrm{w})$ \\
\hline 12917.3 & $* *$ & $* * *(\mathrm{bl})$ & $* * *$ & $*$ & $\mathrm{Na}, \mathrm{H}_{2} \mathrm{O}, \mathrm{FeH}(\mathrm{wk})$ \\
\hline 12920.0 & $*$ & $*$ & $*$ & $* * *(\mathrm{spl})$ & $\mathrm{Ti}, \mathrm{H}_{2} \mathrm{O}, \mathrm{FeH}(\mathrm{wk})$ \\
\hline 12922.1 & $*$ & $* *$ & $* *$ & $* *(\mathrm{spl})$ & $\mathrm{Cr}, \mathrm{FeH}$ \\
\hline 12923.9 & $*$ & $*$ & $*$ & $*$ & $\mathrm{H}_{2} \mathrm{O}, \mathrm{FeH}$ \\
\hline 12925.7 & $* *$ & $* * *$ & $* *$ & $*$ & $\mathrm{FeH}(?), \mathrm{H}_{2} \mathrm{O}(?)$ \\
\hline 12929.6 & $* *$ & $* * *$ & $* *$ & $*$ & $\mathrm{FeH}, \mathrm{H}_{2} \mathrm{O}$ \\
\hline 12932.5 & $* * *$ & $* * *$ & $* * *$ & ** & $\mathrm{H}_{2} \mathrm{O}, \mathrm{FeH}(\mathrm{w})$ \\
\hline 12934.6 & $*$ & $*$ & $*$ & * & $\mathrm{FeH}, \mathrm{H}_{2} \mathrm{O}, \mathrm{CrH}(\mathrm{w})$ \\
\hline 12937.0 & $*$ & $*$ & $*$ & $* * *(\mathrm{spl})$ & $\mathrm{Cr}, \mathrm{H}_{2} \mathrm{O}$ \\
\hline 12939.5 & $* *$ & $* * *$ & $* * *$ & $*$ & $\mathrm{FeH}$ \\
\hline 12941.7 & $* *$ & $* *$ & $* *$ & $* *$ & $\mathrm{FeH}(\mathrm{w}), \mathrm{H}_{2} \mathrm{O}(?)$ \\
\hline 12943.8 & $* *$ & $* * *$ & $* *$ & $*$ & $\mathrm{FeH}, \mathrm{CrH}(\mathrm{w}), \mathrm{H}_{2} \mathrm{O}$ \\
\hline 12945.4 & $*$ & $* *$ & $* *$ & $*$ & $\mathrm{FeH}, \mathrm{H}_{2} \mathrm{O}(?)$ \\
\hline 12951.0 & $* *$ & $* * *$ & $* * *$ & $* *$ & $\mathrm{CrH}, \mathrm{H}_{2} \mathrm{O}$ \\
\hline 12952.7 & $* *$ & $* *$ & $* *$ & $* *$ & $\mathrm{H}_{2} \mathrm{O}(?), \mathrm{FeH}(\mathrm{w})$ \\
\hline 12953.8 & $* *$ & $* * *$ & $* * *$ & $*$ & $\mathrm{H}_{2} \mathrm{O}$ \\
\hline 12955.9 & $* *$ & $* * *$ & $* * *$ & $* *$ & $\mathrm{H}_{2} \mathrm{O}, \mathrm{FeH}(\mathrm{w})$ \\
\hline 12957.2 & $* *$ & $* *$ & $* * *$ & $* *$ & $\mathrm{H}_{2} \mathrm{O}$ \\
\hline 12959.6 & $* *$ & $* * *$ & $* * *$ & $* *$ & $\mathrm{H}_{2} \mathrm{O}(?)$ \\
\hline 12962.3 & $* *$ & $* *$ & $* *$ & $* *$ & $\mathrm{H}_{2} \mathrm{O}$ \\
\hline 12964.2 & $*$ & $* *$ & $* *$ & $* *$ & $\mathrm{FeH}(\mathrm{w}), \mathrm{H}_{2} \mathrm{O}$ \\
\hline 12966.6 & ** & $* * *$ & $* * *$ & $* *$ & $\mathrm{H}_{2} \mathrm{O}, \mathrm{FeH}(\mathrm{w})$ \\
\hline 12968.4 & $*$ & $*$ & $* *$ & $*$ & $\mathrm{H}_{2} \mathrm{O}$ \\
\hline 12969.9 & $* *$ & $* * *$ & $* * *$ & $*$ & $\mathrm{H}_{2} \mathrm{O}(?)$ \\
\hline 12973.9 & $*$ & - & $* *$ & $*$ & $\mathrm{FeH}$ \\
\hline 12975.2 & $* *$ & $* *$ & $* *$ & $*(\mathrm{bl})$ & $\mathrm{H}_{2} \mathrm{O}$ \\
\hline 12975.9 & $* *$ & $* *$ & $* *$ & $* * *(\mathrm{spl})$ & $\mathrm{Mn}, \mathrm{H}_{2} \mathrm{O}$ \\
\hline 12977.5 & $*$ & $* *$ & $* *$ & $*$ & $\mathrm{H}_{2} \mathrm{O}, \mathrm{FeH}(\mathrm{wk})$ \\
\hline 12978.7 & $*$ & $* *$ & $* *$ & $* *$ & $\mathrm{H}_{2} \mathrm{O}(?), \mathrm{FeH}(\mathrm{w})$ \\
\hline 12980.8 & $* * *$ & $* * *$ & $* * *$ & $*$ & $\mathrm{CrH}, \mathrm{H}_{2} \mathrm{O}, \mathrm{FeH}(\mathrm{w})$ \\
\hline 12982.2 & $*$ & $*$ & $*$ & $*$ & $\mathrm{H}_{2} \mathrm{O}(?)$ \\
\hline 12988.9 & $*$ & $* *$ & $*$ & $*$ & $\mathrm{H}_{2} \mathrm{O}$ \\
\hline
\end{tabular}

2700/5.0/0.0 synthetic one with $v \sin i \sim 10 \mathrm{~km} \mathrm{~s}^{-1}$ (e.g., Jones et al. 2005). For both observed spectra the positions of atomic lines in the synthetic spectra correspond to lines in the observed spectrum though intensities of computed and observed lines differ significantly for some lines. Many lines are blended by molecular lines of approximately the same strength. For example, the Fe I $12879.76 \AA$ line profile is described well in the Arcturus spectrum (see Lyubchik et al. 2004) but could not be fitted in our spectra because of severe blending by molecular lines. 
Table 2. continued.

\begin{tabular}{cccccl}
\hline \hline WL $\pm 0.1 \AA$ air & GJ406 & VB10 & 2M0140+27 & Sunspot & Notes \\
\hline 12990.8 & $*$ & $* * *$ & $* *$ & - & $\mathrm{FeH}, \mathrm{H}_{2} \mathrm{O}$ \\
12993.2 & $*$ & $*$ & $*$ & $*$ & $\mathrm{H}_{2} \mathrm{O}, \mathrm{CrH}(\mathrm{w}), \mathrm{FeH}(\mathrm{w})$ \\
12996.3 & $*$ & $* *$ & $*$ & $*$ & $\mathrm{H}_{2} \mathrm{O}(?)$ \\
12998.4 & $* *$ & $* * *$ & $* *$ & $*$ & $\mathrm{FeH}, \mathrm{H}_{2} \mathrm{O}$ \\
13000.1 & $*$ & $* * *$ & $*$ & $*$ & $\mathrm{H}_{2} \mathrm{O}(?), \mathrm{FeH}(?)$ \\
13000.7 & $*$ & $* * *$ & $*$ & - & $\mathrm{H}_{2} \mathrm{O}$ \\
13001.4 & - & - & - & $* *(\mathrm{spl})$ & $\mathrm{Ca}$ \\
13001.9 & $*$ & $* * *$ & $*$ & $* *$ & $\mathrm{H}_{2} \mathrm{O}$ \\
\hline
\end{tabular}

Asterisks correspond to different spectral line intensities:

$*-0.96-0.99, * *-0.91-0.95, * * *-<0.95$ (see text).

$\mathrm{bl}$ - the line feature is blended by other features.

$\mathrm{w}-$ the wing of the line.

wk - (very) weak line.

? - This species probably forms the line-feature.

$\mathrm{spl}$ - the line is split by magnetic field (solar lines).

VALD - Vienna Atomic Line Database (Kupka et al. 1999).

“-" not identified.

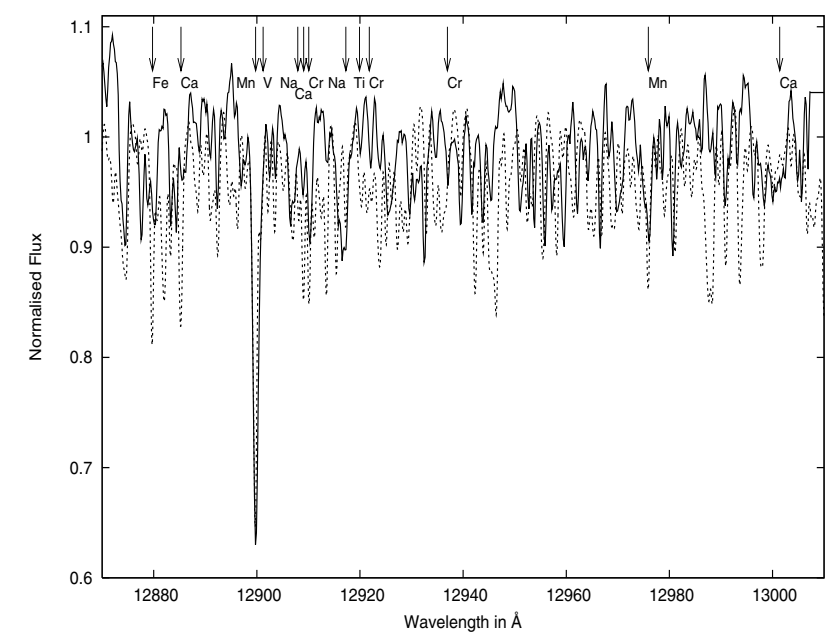

Fig. 4. The observed spectrum of GJ406 (M6 V) (solid line) compared to a $2800 / 5.0 / 0.0, v \sin i=3 \mathrm{~km} \mathrm{~s}^{-1}$ synthetic spectrum using molecular line lists of $\mathrm{H}_{2} \mathrm{O}(\mathrm{BT} 2)$, $\mathrm{TiO}$ and $\mathrm{FeH}$ (dotted line).

\section{2. $2 \mathrm{M} 0140+27$}

In Fig. 6 the observed spectrum of 2M0140+27 (M8.5 V) is compared to a synthetic spectrum 2500/5.0/0.0 with $v \sin i=6.5 \mathrm{~km} \mathrm{~s}^{-1}$ (based on Reid et al. 2002). The Mn I $12899.76 \AA$ line is well fit, though the fit to Fe and other weak atomic lines is not good at all. The rotational velocity is similar to the observational resolution and thus does not significantly change the synthetic line profiles. Thus $2 \mathrm{M} 0140+27$ also provides a good template for the identification of lines and their analysis.

\section{3. $L P 944-20$}

LP944-20 is a fast rotating dwarf with $v \sin i \sim 30 \mathrm{~km} \mathrm{~s}^{-1}$ (e.g., Tinney \& Reid 1998). In Fig. 7 we present observations of the brown dwarf LP 944-20 (M9 V/M9.5 V) with a 2400/4.5/0.0 synthetic spectrum with $v \sin i=30 \mathrm{~km} \mathrm{~s}^{-1}$. We find the large rotational broadening restricts the accuracy of our analysis. Only stronger lines like Mn 12899.76 A can be reliably identified in the observed spectrum. In general, the synthetic spectra does not

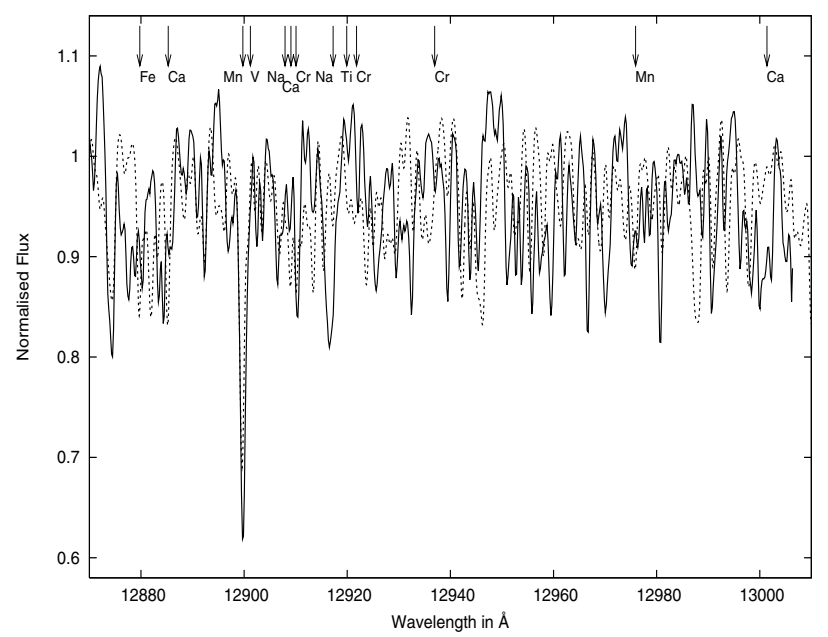

Fig. 5. The observed VB 10 (M8 V) spectrum (solid line) compared to a 2700/5.0/0.0, $v \sin i=10 \mathrm{~km} \mathrm{~s}^{-1}$ synthetic spectrum with molecular line lists of $\mathrm{H}_{2} \mathrm{O}$ (BT2), $\mathrm{TiO}$ and $\mathrm{FeH}$ (dotted line).

particularly resemble the observed one. This may be partially explained by the effects of chromospheric-like structures, vertical stratification in the outermost layers of LP944-20 (see Pavlenko et al. 2006b) or even abundance peculiarities.

The poor match of FeH absorption seems key. In the synthetic spectrum we see some "features" created by the synthetic FeH absorption at $12880,12916,12947,12988 \AA$, which are absent in the observed spectrum. Features at these wavelengths can be recognized also in the VB10 spectrum. However, given that observed $\mathrm{FeH}$ features should increase toward lower temperatures in late M-dwarfs (Kirkpatrick et al. 1999; Cushing et al. 2005) we suspect these discrepancies are caused by FeH line list inaccuracies.

\section{4. $2 M 0345+25$}

The lines in the spectrum of the L0 dwarf $2 \mathrm{M} 0345+25$ are very broad (see Fig. 8). We fit the observed spectrum of 2M0345+35 with a $2200 / 5.0 / 0.0$ synthetic spectra $\left(v \sin i=45 \mathrm{~km} \mathrm{~s}^{-1}\right)$. The $v \sin i$ value was determined by the synthetic spectra fit to the 


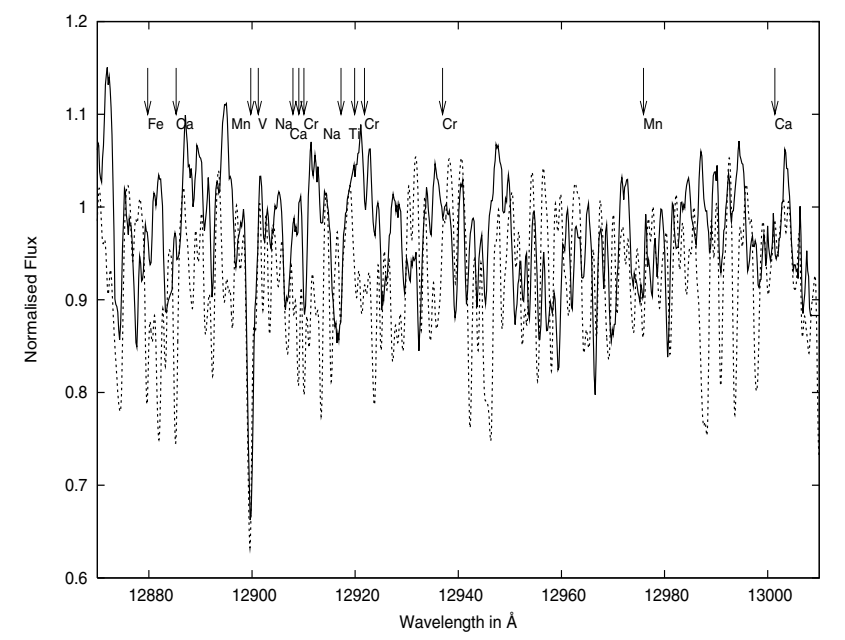

Fig. 6. The observed $2 \mathrm{M} 0140+27(\mathrm{M} 8.5 \mathrm{~V})$ spectrum is compared to the $2500 / 5.0 / 0 / 0, v \sin i=6.5 \mathrm{~km} \mathrm{~s}^{-1}$ synthetic spectrum with molecular line lists of $\mathrm{H}_{2} \mathrm{O}$ (BT2), TiO, $\mathrm{FeH}$ (dotted line).

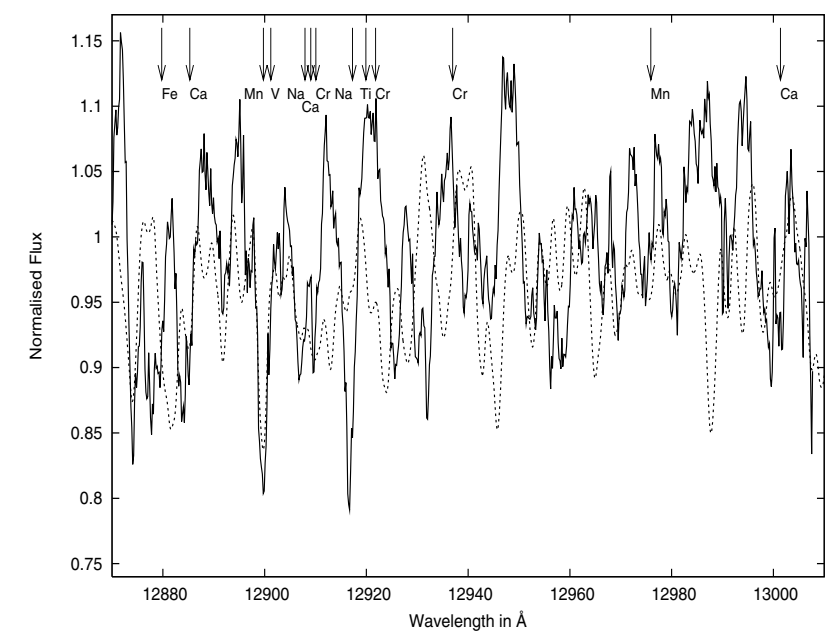

Fig. 7. The observed LP944-20 (M9 V) spectrum (solid line) compared with the $2400 / 4.5 / 0.0, v \sin i=30 \mathrm{~km} \mathrm{~s}^{-1}$ synthetic spectrum with molecular line lists of $\mathrm{H}_{2} \mathrm{O}(\mathrm{BT} 2), \mathrm{TiO}, \mathrm{FeH}$ (dotted line).

wings of Mn I $12899.76 \AA$ line in the observed spectrum. The strong Mn I $12899.76 \AA$ A line profile fits satisfactorily. Other atomic features, including $\mathrm{Fe}$, seem to disappear under the strong pseudo-continuum formed by molecular lines. Again, as with LP944-20, the synthetic spectra including FeH predict a number of features in the synthetic spectrum which are not observed.

\subsection{Line identification}

Based on the observable spectra of three M-dwarfs which are less affected by rotation (GJ406, VB10 and 2M0140+27), we make identifications of absorption lines. These identifications are performed by comparison of each observed spectra with synthetic ones calculated for each molecular line list of the principal contributors to opacity in this region.

Results of these identifications are presented in Table 2 . We classify the absorption line-like features in three groups based on their intensity: one asterisk $\left(^{*}\right)$ in Cols. 2-4 of Table 2 corresponds to a mean "residual intensity" of the absorption line from 0.96-0.99 (where a value of 1 corresponds to the level of pseudo-continuum, not the real continuum

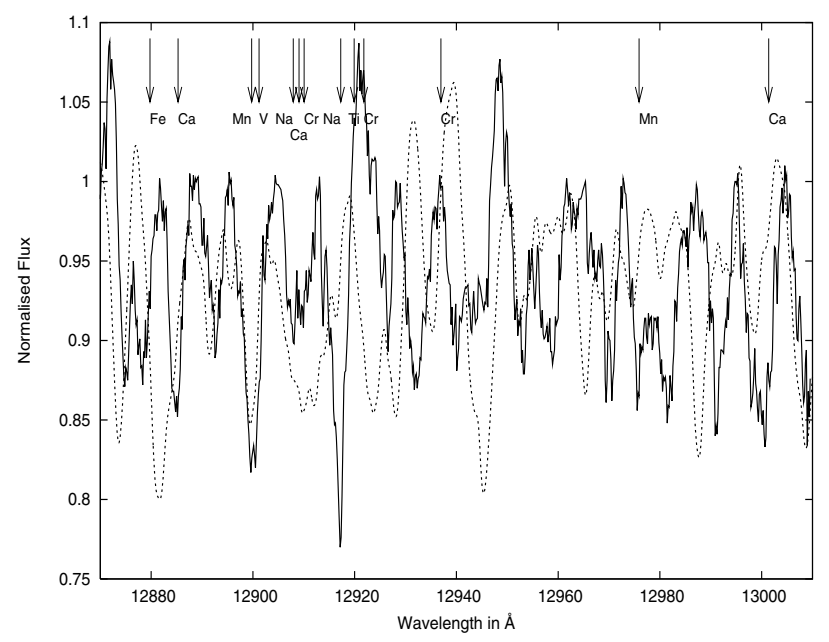

Fig. 8. The observed $2 \mathrm{M} 0345+25$ (L0) (solid line) spectrum is compared to the 2200/5.0/0.0, $v \sin i=45 \mathrm{~km} \mathrm{~s}^{-1}$ synthetic spectrum computed using molecular line lists of $\mathrm{H}_{2} \mathrm{O}$ (BT2), $\mathrm{TiO}, \mathrm{FeH}$ (dotted line).

level); two asterisks (**) corresponds to a "residual intensity" between 0.91-0.95; three asterisks (***) labels absorption lines deeper than 0.90 . We also check for these lines in the solar umbra spectrum (Wallace \& Livingston 1992, ftp://ftp.noao.edu/fts/spot1atl) (Col. 5), asterisks mean the same as above. Thus in Col. 6 of Table 2 we identify species, which form line-features in the observed spectrum, however, a number of synthetic lines are located at toodiscrepant wavelengths or are too weak to make the observed spectral features. In these cases we put a "?" to signify the uncertainty in their identification.

As the next step in line identification we use lines, identified in Table 2, with residual intensities larger than 0.9 (three asterisks in Cols. 2, 3 or 4), to find corresponding line-like features in the spectra of the other two dwarfs: LP944-20 and 2M0345+25, with highly broadened spectral features. These results are shown in Table 3. Despite the large differences in intensity between observed and calculated lines for all features other than $\mathrm{Mn}$ some of the strong synthetic features are present in the observed data and can be identified with confidence.

\subsection{Mn I lines}

Mn I lines form clear features in the spectra of ultra cool dwarfs in the $J$ band, which is relatively free from distinctive molecular absorption bands. In Lyubchik et al. (2004) we make detections using the VALD line list (Kupka et al. 1999; e.g. Kurucz's Mn I data), 11 lines of Mn I lie in the region 12 800-14000. Three of these lines are predicted to be rather strong in the observed spectra of ultra cool dwarfs across the wavelength range of our observed spectra so we try to analyse these lines, however, only the $12899.76 \AA$ line is clearly detected in all the observational spectra. The others are camouflaged and blended by molecular lines. The quality of three spectra allow us to measure the "pseudo equivalent width" (pEW) of the $12899.76 \AA$ line. We note that these values are not the real equivalent width because of uncertain continuum level determination (Pavlenko 1997). Using DECH20 package (Galazutdinov 1992) we determine $\mathrm{pEW}(\mathrm{Mn} \mathrm{I})=501 \mathrm{~m} \AA, 500 \mathrm{~m} \AA$, and $502 \mathrm{~m} \AA$ for GJ406, VB10 and $2 \mathrm{M} 0140+27$, respectively. The accuracy of pseudo equivalent width determinations is $\sim 5 \%$. 
Table 3. Line identifications and strengths for the programme stars LP944-20 and 2M0345+25 are tabulated.

\begin{tabular}{|c|c|c|c|}
\hline $\mathrm{WL} \pm 0.3 \AA$, air & LP944-20 & $2 \mathrm{M} 0345+25$ & Notes \\
\hline 12874.4 & $* * *$ & $* * *$ & $\mathrm{FeH}, \mathrm{H}_{2} \mathrm{O}(\mathrm{bl})$ \\
\hline 12877.7 & $* * *$ & $* * *$ & $\mathrm{FeH}(?), \mathrm{TiO}(\mathrm{wk}, ?), \mathrm{H}_{2} \mathrm{O}(?)$ \\
\hline 12880.4 & $* * *$ & $-(\mathrm{bl})$ & $\mathrm{H}_{2} \mathrm{O}, \mathrm{FeH}(?)$ \\
\hline 12883.5 & $* * *$ & $-(\mathrm{bl})$ & $\mathrm{FeH}, \mathrm{TiO}$ (wk) \\
\hline 12884.4 & $* * *$ & $* * *$ & $\mathrm{FeH}(\mathrm{w}), \mathrm{H}_{2} \mathrm{O}(\mathrm{wk})$ \\
\hline 12885.3 & $* * *$ & $* * *$ & $\mathrm{Ca}$ (VALD), $\mathrm{FeH}$ \\
\hline 12892.4 & $* * *$ & $* *$ & $\mathrm{H}_{2} \mathrm{O}, \mathrm{FeH}, \mathrm{TiO}(w k)$ \\
\hline 12899.8 & $* * *$ & $* * *$ & Mn (VALD) \\
\hline 12906.5 & $* * *$ & $* *$ & $\mathrm{H}_{2} \mathrm{O}, \mathrm{FeH}(\mathrm{w}), \mathrm{TiO}(\mathrm{wk})$ \\
\hline 12907.9 & $* * *(\mathrm{bl})$ & $* *$ & $\mathrm{Na}$ (VALD) \\
\hline 12910.4 & - & - & $\mathrm{CrH}, \mathrm{H}_{2} \mathrm{O}, \mathrm{FeH}$ \\
\hline 12916.5 & $* * *$ & $* * *(\mathrm{bl})$ & $\mathrm{H}_{2} \mathrm{O}, \mathrm{FeH}(\mathrm{w}), \mathrm{TiO}(\mathrm{w})$ \\
\hline 12917.3 & $* * *$ & $* * *$ & $\mathrm{Na}, \mathrm{H}_{2} \mathrm{O}, \mathrm{FeH}(\mathrm{wk})$ \\
\hline 12925.7 & $* * *$ & $*$ & $\mathrm{FeH}(\mathrm{w}), \mathrm{H}_{2} \mathrm{O}(?)$ \\
\hline 12929.6 & $* * *$ & - & $\mathrm{FeH}(\mathrm{w}), \mathrm{H}_{2} \mathrm{O}(?)$ \\
\hline 12932.5 & $* * *(\mathrm{bl})$ & $* *(\mathrm{bl})$ & $\mathrm{H}_{2} \mathrm{O}, \mathrm{FeH}(\mathrm{w})$ \\
\hline 12939.5 & $* * *$ & $*$ & $\mathrm{FeH}$ \\
\hline 12943.6 & - & - & $\mathrm{FeH}, \mathrm{CrH}, \mathrm{H}_{2} \mathrm{O}$ \\
\hline 12951.0 & $* * *$ & $-(\mathrm{bl})$ & $\mathrm{CrH}, \mathrm{H}_{2} \mathrm{O}, \mathrm{FeH}$ \\
\hline 12953.8 & - & $* *(\mathrm{bl})$ & $\mathrm{H}_{2} \mathrm{O}, \mathrm{FeH}$ \\
\hline 12955.9 & $* * *$ & - & $\mathrm{H}_{2} \mathrm{O}, \mathrm{FeH}(\mathrm{w})$ \\
\hline 12957.2 & $* * *$ & $* *(\mathrm{bl})$ & $\mathrm{H}_{2} \mathrm{O}$ \\
\hline 12959.6 & $* * *(\mathrm{bl})$ & $* *$ & $\mathrm{H}_{2} \mathrm{O}(?)$ \\
\hline 12966.6 & $* * *$ & - & $\mathrm{H}_{2} \mathrm{O}, \mathrm{FeH}(\mathrm{w})$ \\
\hline 12969.7 & $* * *$ & $* * *$ & $\mathrm{H}_{2} \mathrm{O}$ \\
\hline 12980.8 & $* * *$ & $* *$ & $\mathrm{H}_{2} \mathrm{O}, \mathrm{CrH}, \mathrm{FeH}$ \\
\hline 12990.8 & $* * *$ & $* * *$ & $\mathrm{FeH}, \mathrm{H}_{2} \mathrm{O}$ \\
\hline 12998.4 & $* * *(\mathrm{bl})$ & $* * *(\mathrm{bl})$ & $\mathrm{FeH}, \mathrm{H}_{2} \mathrm{O}, \mathrm{CrH}(\mathrm{w})$ \\
\hline 13000.1 & $* * *$ & $* * *(\mathrm{bl})$ & $\mathrm{H}_{2} \mathrm{O}(?)$ \\
\hline 13000.7 & $* * *$ & $* * *$ & $\mathrm{H}_{2} \mathrm{O}$ \\
\hline 13001.9 & $* * *$ & $* * *$ & $\mathrm{H}_{2} \mathrm{O}$ \\
\hline
\end{tabular}

Asterisks correspond to different spectral line intensities: * - 0.96-0.99, ** - 0.91-0.95,*** $-<0.95$ (see text);

bl - the line feature is blended by other features; $\mathrm{w}$ - the wing of the line;

wk - (very) weak line;

? - this species probably forms the line-feature;

$\mathrm{spl}$ - the line is split by magnetic field (solar lines);

VALD - Vienna Atomic Line Database (Kupka et al. 1999);

"_" not identified.

Table 4. $J$-band Mn I lines basic parameters.

\begin{tabular}{cccc}
\hline \hline Wavelength, $\AA$ & $\chi, \mathrm{eV}$ & $\log g f, \mathrm{VALD}$ & $\log g f^{\star}, \mathrm{Me} 99$ \\
\hline 12899.764 & 2.114 & -1.059 & -1.76 \\
12975.912 & 2.889 & -0.937 & -1.57 \\
\hline
\end{tabular}

VALD - Kupka et al. (1999); Me99 - Melèndez (1999).

* Value for the main hyperfine component.

Melèndez (1999) and Holt et al. (1999) indicate that the profiles of Mn lines and abundance determination depend on the hyperfine structure (HFS) and it is thus important to consider HFS for accurate abundance analysis. In Table 4 the available parameters of the Mn I lines used in computations are presented. Importantly an experimental $\log g f$ value of the $12899.76 \AA$ An I line obtained by Imperial College group (UK) is close to the VALD value (Blackwell-Whitehead et al. private communication).

The synthetic spectra to examine the HFS of Mn I are computed using NextGen model atmospheres (Hauschildt et al. 1999 ) with parameters $T_{\text {eff }}=2800 \mathrm{~K}, \log g=5.0,[\mathrm{M} / \mathrm{H}]=0.0$.

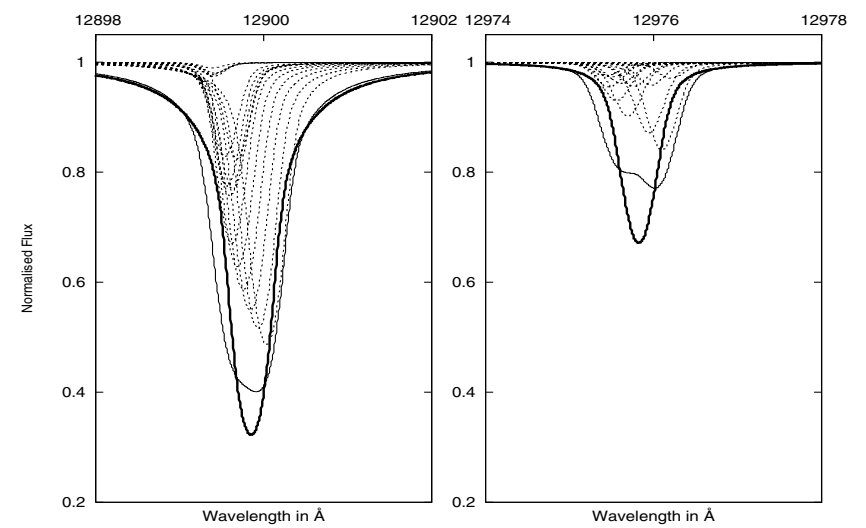

Fig. 9. Computations of $\mathrm{Mn}$ I line profiles with and without hyperfine splitting: the thick solid line - VALD log gf for Mn I, thin solid line computations with all hyper-fine components (Melèndez 1999), dashed lines - computations for each hyper-fine component of Mn I line.

The data on wavelengths and relative intensities of hyperfine components for computations are taken from Melèndez (1999). From Fig. 9 it is seen that line profiles computed using the VALD data and the average of HFS curve do not differ in a drastic way for Mn I 12 899.76 ̊ and $12975.91 \AA$ lines. However, our problems with making reliable line identifications mean that before HFS effects are reliably diagnosed at these resolutions it will be necessary to get a more reliable fit to the bulk of weaker features which are providing the pseudo-continuum.

\section{Discussion}

In the case of the strong Mn lines, where we have reliable knowledge of the oscillator strengths we find satisfactory fits. This indicates that the overall level of opacity is reasonably well described. However, apart from the Mn lines we have found a rather poor match between our observations and synthetic spectra across the 12810-13010 A region. We are not aware of high resolution spectral analysis using recent water line lists in this region, however, the previous good fits have been obtained with observations at longer wavelengths (e.g., Jones et al. 2005) and our knowledge of the line list construction mean that we have confidence that water is reasonably well described over this region. Furthermore, in our calculations we used two different line lists of $\mathrm{H}_{2} \mathrm{O}$ : AMES (Partridge \& Schwenke 1997) and BT2 (Barber et al. 2006) and our inter-comparison of these line lists indicate that differences between them in the 12810-13010 are not significant for this analysis. According to our opacity calculations molecular opacities other than water, namely $\mathrm{FeH}$ and $\mathrm{CrH}$, are important for an adequate modelling of the 12 810-13010 A region. However, the features predicted for these $\mathrm{FeH}$ and $\mathrm{CrH}$ line lists are not clearly evident. We thus suspect that the lack of overall match is due primarily to problems with these line lists.

With the exception of the Mn line our fits between observed and synthetic spectra is poor. Nonetheless, the quality of other atomic opacities are difficult to judge since they are too weak to reliably distinguish from the pseudo-continuum. Thus the poor fits of the weak atomic lines cannot be considered as a product of poorly known atomic parameters. Furthermore, across all of the temperature range considered for our observed spectra we expect and observe blends formed by atomic and molecular lines. It thus seems that further computations and/or measurements of molecular line lists for $\mathrm{FeH}$ and $\mathrm{CrH}$ will be necessary in order to 
carry out $J$ band spectral synthesis of ultra cool dwarfs. Without such line lists spectral analysis of atomic features will be limited to a very small number of strong lines.

In our analysis we did not use dusty model atmospheres. Many authors have stressed that dust is important for atmospheric temperatures <2700 K (e.g., Jones \& Tsuji 1997). However, comparisons of synthetic spectra computations using dusty models (Allard 2005) and non-dusty ones (Hauschildt et al. 1999) show that dust effects are considerably lower than uncertainties in line lists of molecules. Furthermore, dust opacity is modelled to produce a smooth opacity in a relatively high resolution study such as this. If dust is the dominant opacity across this region we would not anticipate to be able to fit the Mn line at $12899.76 \AA$.

Acknowledgements. We would like to thank the authors of the PHOENIX model atmospheres and the Sunspot umbra atlas for making their data available through their ftp sites. The NSO/Kitt Peak FTS data used here were produced by NFS/NOAO. The authors thank the Royal Society and PPARC for support of travel and experimental work.

\section{References}

Allen, P. R., Koerner, D. N., Reid, I. N., \& Trilling, D. E. 2005, ApJ, 625, 385

Allard, F. 2005, in Ultra low mass star formation and evolution, ed. A. Magazzu, \& E. Martin, in press

Anders, E., \& Grevesse, N. 1989, Geochim Cosmochim. Acta, 53, 197

Barber, R. J., Tennyson, J., Harris, G. J., \& Tolchenov, R. 2006, MNRAS, 368, 1087

Basri, G., Mohanty, S., Allard, F., et al. 2000, ApJ, 538, 363

Bauschlicher, C. W., Jr., Ram, R. S., Bernath, P. F., Parsons, C. G., \& Galehouse, D. 2001, J. Chem. Phys., 115, 1312

Burrows, A., Ram, R. S., Bernath, P., Sharp, C. M., \& Milson, J. A. 2002, ApJ, 577,986

Cushing, M. C., Rayner, J. T., \& Vacca, W. D. 2005, ApJ, 623, 1115

Dulick, M., Bauschlicher, C. W., Jr., Burrows, A., et al. 2003, ApJ, 594, 651

Galazutdinov, G. A. 1992, Preprint SAO RAN, 92

Gizis, J. E., Monet, D. G., Reid, I. N., et al. 2000, AJ, 120, 1085

Golimowski, D. A., Legget, S. K., Marley, M. S., et al. 2004, AJ, 127, 3516

Gray, D. F. 1976, The observation and analysis of stellar photospheres (New York: Wiley-Interscience), 484

Hauschildt, P. H., Allard, F., \& Baron, E. 1999, ApJ, 512, 377

Henry, T. J., Jao, W., Subasavage, J. P., et al. 2005, ASP Conf. Ser., 338, 228

Holt, R. A., Scholl, T. J., \& Rosner, S. D. 1999, MNRAS, 306, 107

Jones, H. R. A., \& Tsuji, T. 1997, ApJ, 480, L39

Jones, H. R. A., Longmore, A. J., Jameson, R. F., \& Mountain, C. M. 1994, MNRAS, 267, 413

Jones, H. R. A., Longmore, A. J., Allard, F., \& Hauschildt, P. H. 1996, MNRAS, 280, 77
Jones, H. R. A., Pavlenko, Ya., Viti, S., \& Tennyson, J. 2002, MNRAS, 330, 675 Jones, H. R. A., Pavlenko, Ya., Viti, S., \& Tennyson, J. 2003, in 12th Cambridge Workshop on Cool Stars, Stellar Systems, and the Sun (2001 July 30-August 3), ed. A. Brown, G. M. Harper, \& T. R. Ayres (University of Colorado), 899

Jones, H. R. A., Pavlenko, Ya., Viti, S., et al. 2005, MNRAS, 358, 105

Jrgensen, U. G., Jensen, P., Sorensen, G. O., \& Aringer, B. 2001, A\&A, 372, 249

Kirkpatrick, J. D., Henry, T. J., \& McCarthy, D. W. Jr. 1991, ApJS, 77, 417

Kirkpatrick, J. D., Kelly, D. M., Rieke, G. H., et al. 1993, ApJ, 402, 643

Kirkpatrick, J. D., Reid, N. I., Liebert, J., et al. 1999, ApJ, 519, 802

Kupka, F., Piskunov, N. E., Ryabchikova, T. A., Stempels, H. C., \& Weiss, W. W. 1999, A\&AS, 138, 119

Kurucz, R. L. 1994, CD-ROM No.19

Leggett, S. K., Allard, F., Geballe, T. R., Hauschildt, P. H., \& Schweitzer, A. 2001, ApJ, 548, 908

Lyubchik, Yu., Jones, H. R. A., Pavlenko, Ya. V., et al. 2004, A\&A, 416, 655

Martin, E. L., Guenther, E., Zapatero Osorio, M. R., et al. 2006, ApJ, 644, 75

McLean, I. S., McGovern, M. R., Burgasser, A. J., et al. 2003, ApJ, 596, 561

McLean, I. S., Prato, L., McGovern, M. R., et al. 2007, ApJ, 658, 1217

Melèndez, J. 1999, MNRAS, 307, 197

Miller, S., Tennyson, J., Jones, H. R. A., \& Longmore, A. J. 1994, in IAU Coll. 146, ed. U. G. Jrgensen (Berlin: Springer-Verlag), 296

Mohanty, S., \& Basri, G. 2003, ApJ, 583, 451

Murrell, J. N., Carter, S., Farantos, S. C., Huxley, P., \& Varandas, A. J. C. 1984, Molecular Potential Energy Functions (Wiley, Chichester)

Partridge, H., \& Schwenke, D. 1997, J. Chem. Phys., 106, 4618

Pavlenko, Ya. V. 1997, A\&AS, 253, 43

Pavlenko, Ya. V. 2000, Astron. Rep., 44, 219

Pavlenko, Ya.V. 2002, Astron. Rep., 46, 567

Pavlenko, Ya., Zapatero Osorio, M. R., \& Rebolo, R. 2000, A\&A, 355, 245

Pavlenko, Ya. V., Jones, H. R. A., Lyubchik, Yu., Tennyson, J., \& Pinfield, D. J. 2006a, A\&A, 447, 709

Pavlenko, Ya. V., Jones, H. R. A., Martin, E. L., Guenther, E., \& Kenworthy, M. 2006b, MNRAS, accepted

Plez, B. 1998, A\&A, 337, 495

Prato, L., Simon, M., Mazeh, T., Zucker, S., \& McLean, I. S. 2002a, ApJ, 579, L99

Prato, L., Simon, M., Mazeh, T., et al. 2002b, ApJ, 569, 863

Reid, I. N., Kirkpatrick, J. D., Liebert, J., et al. 2002, AJ, 124, 519

Rothman, L. S., Gamache, R. R., Tipping, R. H., et al. 1992, J. Quant. Spectrosc. Radiat. Transf., 48, 469

Shirin, S. V., Polyansky, O. L., Zobov, N. F., Barletta, P., \& Tennyson, J. 2003, J. Chem. Phys., 118, 2124

Schweitzer, A., Hauschildt, P., Allard, F., \& Basri, G. 1996, MNRAS, 283, 821

Schwenke, D. W. 1998, J. Chem. Soc. Faraday Discus., 109, 321

Schwenke, D. W., \& Partridge, H. 2000, J. Chem. Phys., 113, 16

Tennyson, J., Kostin, M. A., Barletta, P., et al. 2004, Comput. Phys. Com., 163, 85

Tinney, C. G., \& Reid, I. N. 1998, MNRAS, 301, 1031

Viti, S., Tennyson, J., \& Polyansky, O. L. 1997, MNRAS, 287, 79

Wallace, L., \& Livingston, W. 1992, An Atlas of a Dark Sunspot Umbral Spectrum from 1970 to $8640 \mathrm{~cm}^{-1}$ (1.16 to $\left.5.1 \mu \mathrm{m}\right)$, NSO Technical Report No. 92-001

Zapatero Osorio, M. R., Martin, E. L., Bouy, H., et al. 2006, ApJ, 647, 1405 